\title{
EVALUATION OF HIDDEN MARKOV MODEL BASED ADAPTIVE PROVISIONING OF OPTICAL BURST SWITCHING NETWORKS AMENABLE FOR UPGRADATION TO GREEN FLEXIGRID NETWORKS
}

\author{
${ }^{1}$ Ramesh, P.G.V. and ${ }^{2}$ Prita Nair \\ ${ }^{1}$ Department of Electronics and Communication, \\ St. Joseph's College of Engineering, Anna University Chennai, India \\ ${ }^{2}$ Department of Physics, Optical Research and Networks Lab, SSN College of Engineering, Chennai, India
}

Received 2013-12-16; Revised 2014-01-10; Accepted 2014-01-16

\begin{abstract}
Catering to the evolving bandwidth-on-demand applications requires flexible provisioning architectures that ensure fairness to both high end and low end users of internet. Dynamic classification of network paths into long and short, based on traffic dependent connection holding times and a wavelength allocation from different subsets for these categories is a necessity for implementing energy saving hybrid switching, loss recovery and flexi grid bit rate variable schemes. Therefore, this study evaluates such a scheme of dynamic classification based on HMM predicted connection-holding times with tightly integrated adaptive burst sizing and segregated wavelength allocation for long and short categories. Simulation study of a 28 node OBS network shows that this coupled scheme reduces delays and results in throughput improvement of $67 \%$ for long and $12 \%$ for short traffic over schemes that employ adaptive burst sizing based on number of active traffic flows and independent wavelength assignment schemes.
\end{abstract}

Keywords: Traffic Prediction, Wavelength Assignment, Adaptive Resource Allocation, Link Holding Time

\section{INTRODUCTION}

Evolving nature of service delivery, fueled by growing bandwidth-on-demand applications and wireless backhaul is forcing the upgradation of modulation formats from simple 10 Gbps OOK per DWDM channel to complex DPSK and multibit QAM modulation formats and is expected to lead ultimately to flexi grid or elastic optical networks right down to the access networks (Gerstel et al., 2012). But these complex schemes though economically feasible in the core networks have to seamlessly integrate with less complex schemes that the legacy metro and access networks can gracefully migrate to, so as to ensure low operational expenses to the end-users. Resource provisioning in Optical Burst Switching (OBS), has to be modified so that they can easily adopt hybrid schemes for loss recovery, hybrid switching and flexible bit-rates.
When paths in the core network have to dynamically handle different types of bursts either long or short in duration, then accordingly optimal schemes also have to be varied dynamically ensuring energy saving and maximum throughput with minimal delay. If in addition, wavelength allocation is also done from within designated wavebands for these different types of traffic, it is also possible to ensure wavelength continuity in critical applications even down to access networks allowing compatibility with legacy systems. This would require provisioning based on dynamic path-dependent connection holding times. If the predicted connection holding time of the chosen path is used for adaptive burst sizing and wavelength allocation, then the blocking probability and burst losses can be minimized. Tornatore et al. (2005) have shown that a knowledge of required connection holding times for any incoming application can ensure resource

Corresponding Author: Ramesh, P.G.V., Department of Electronics and Communication, St. Joseph's College of Engineering, Anna University Chennai, India 
savings through efficient shared-path protection. The work of Fiorani et al. (2013) illustrates that knowledge of the short and long traffic paths in the network is crucial for implementing of energy efficient hybrid switching (Fiorani et al., 2013). Therefore our approach of predicting path-dependent connection-holding times available in the network at any time when matched to the knowledge of requirements of incoming application will ensure efficient transport.

Reports so far show resource allocation schemes for burst sizing and wavelength allocation are carried out mostly independent of each other. They can be broadly classified into service differentiated prioritybased (Zhang et al., 2009; Coulibaly et al., 2011), total TCP flow-level statistics based (Ramantas and Vlachos, 2011; Bonald et al., 2011) or feedback based (Rumley et al., 2009) schemes for adaptive burst sizing and minimum wavelength usage based or statistical usage based (Johnson et al., 2001; Drakos et al., 2012; Shekhawat et al., 2008) schemes for wavelength allocation. Adaptive optical burst sizing schemes reported by Bonald et al. (2011) adjusts the burst sizes based on total number of traffic flows and not on the available path-specific connection times. The closest approach to path dependent connection aware scheduling is the one reported by (Rumley et al., 2009) but is prone to delays since each core node controls the allocation based on feedback from other nodes. Therefore appropriate predictive schemes for path-dependent connection-holding times will be required. Hidden Markov Model (HMM), provides the solution for wide variety of applications such speech tagging and noun-phrase chunking (Chomphan, 2012), is also flexible enough to predict any type of internet traffic (Dainotti et al., 2008) and has been demonstrated for wavelength assignment in WDM networks by Johnson et al. (2001). In this study, we use thresholding of HMM predicted path-dependent connection holding times and show that this connection-time impairment aware provisioning of Burst Sizes and Associated Wavelength Allocation (ABS-WA) can reduce delays and improve throughput. This scheme can also be used for dynamically selecting slow or fast switching, ensuring energy saving and can also indicate the scenarios where varying bit-rates will improve the efficiency of transport.

The forthcoming sections 2 and 3 outlines traffic prediction and resource allocation using HMM and section 4 presents the simulation results of execution of this scheme in a 28 node mesh topology.

\section{HMM BASED HOLDING TIME PREDICTION}

In the HMM used in this work, the state of the nodes at any instant of time $t$ in the network form a set, the departure time of a burst, the wavelength assigned to it, the arrival time of a incoming burst and its assigned wavelength are the observations associated with each state. Each possible path between any source to destination pair can be represented as a sequence of transitions of states $\mathrm{C}$ of appropriate length and pathdependent connection holding time for traffic flow between each source destination pairs is modeled as the hidden states of the HMM (Khanna and Liu, 2006). For every observatory period $\mathrm{Tb}$, the difference between departure time from the previous node to the arrival time at the successive hops is estimated from the observables of the system. In this analysis, we assume that the future connection arrival instants and holding times are not known beforehand.

Connection holding time of a burst $\mathrm{km}$ routed on a wavelength $\lambda \mathrm{m}$ through a link $\mathrm{Lij}(\mathrm{km})$ between a pair of nodes $(\mathrm{Ni}, \mathrm{Nj})$, is designated as $\mathrm{CTh}(\mathrm{Lij}(\mathrm{km}))$ and is defined as the difference between the observed time of departure $\mathrm{Td}(\mathrm{km}, \mathrm{Ni})$ of the burst from the transmitting node $\mathrm{Ni}$ to the time of receipt of the entire burst $\mathrm{Ta}(\mathrm{km}, \mathrm{Nj})$ at the receiving node $\mathrm{Nj}$ as shown below in Equation (1):

$\operatorname{CTh}\left(\mathrm{L}_{\mathrm{ij}}\left(\mathrm{k}_{\mathrm{m}}\right)\right)=\operatorname{Ta}\left(\mathrm{km}, \mathrm{N}_{\mathrm{j}}\right)-\operatorname{Td}\left(\mathrm{k}_{\mathrm{m}}, \mathrm{N}_{\mathrm{i}}\right)$

Therefore the connection holding time for the burst $\mathrm{km}$ through the entire path $\mathrm{p} 1$ from source $\mathrm{s}$ to destination d CThsd (km, p1) is the accumulated delay through the multiple intermediate hops between the nodes in the path and can be written as a summation of the connection holding times over all the links in the path as depicted in Equation (2):

$$
\left.\operatorname{CThsd}\left(\mathrm{k}_{\mathrm{m}}, \mathrm{pl}\right)\right)=\sum_{\mathrm{ij}} \operatorname{CTh}\left(\mathrm{L}_{\mathrm{ij}}\left(\mathrm{k}_{\mathrm{m}}\right)\right)
$$

Following the formulation of HMM as per Rabiner (1989), transition array A, storing probability of state $\mathrm{j}$ following state $\mathrm{i}$ (independent of time), observation array $\mathrm{B}$, storing probability of observation $\mathrm{v} 1$ being produced from state $\mathrm{j}$ (independent of time) and $\pi$ the initial probability array are computed. Decoding the HMM to extract the hidden states for a given state sequence is done using Viterbi algorithm. 


\section{RESOURCE ALLOCATION USING HMM}

\subsection{Adaptive burst sizing}

For an incoming application at the ingress node, the HMM predicted connection holding time for the shortest path to the required destination is used to assign burst size adaptively as per ABS-WA algorithm given below. If the predicted link holding time is less than a specified threshold, a minimum burst size is assigned otherwise a burst length less than or equal to a maximum value is assigned:

1: $\mathrm{L}_{\mathrm{h}}=$ the predicted link holding time of a link

2: $\operatorname{minT}_{\mathrm{Lh}}=$ the threshold that defines the minimum link holding time

3: $\min _{\text {size }}=$ the minimum burst size

4: $\max _{\text {size }}=$ the maximum burst size

5: If $\mathrm{L}_{\mathrm{h}} \leq \operatorname{minT}_{\mathrm{Lh}} \quad$ Then

6: Burst Size $=\min _{\text {size }}$

Else $\mathrm{L}_{\mathrm{h}}>\min _{\mathrm{Lh}}$ Then

End if

7: Burst Size $\leq \max _{\text {size }}$

\subsection{Wavelength Allocation}

The proposed wavelength allocation algorithm allocates wavelength to the paths based on its connection holding time. The link holding time differs greatly from a link to another. In section 3.1, our approach classifies the link as either short term or long term based on link holding time. Long-term paths have greater priority on wavelength resources as it has long link holding time. On the other hand, short-term paths have lower priority over resources. Two threshold values Thmin and Thmax are set based on predicted link holding times. The available $\mathrm{N}$ data channels are split in two categories. All wavelength numbers higher than a specific minimum called $\mathrm{Th}_{\text {min }}$ are assigned to short-term paths and all wavelength numbers equal to or less than a maximum called $\mathrm{Th}_{\max }$ is assigned to long traffic. All short-term paths are assigned wavelength channel numbers higher than this threshold number. This wavelength assignment section of ABS-WA algorithm is given below:

1: $\mathrm{Th}_{\min }=$ threshold value representing the minimum wavelength num berfor short- term paths

2: $\mathrm{Th}_{\max }=$ threshold value representing the maximum wavelength number for long-termtraffic

3: If $\left(\mathrm{L}_{\mathrm{h}} \leq \operatorname{minT}_{\mathrm{Lh}}\right)($ connection holding time $=$ Short $)$
Else $\{$ connection holding time $=$ Long $\}$

4: If (connection holding time $=$ Short) then

5: Allocate wavelength $>$ Thmin but $\leq \mathrm{N}$ using LAUC

6: Else (connection holding time $=$ long) then

7: Allocate wavelength $\leq \mathrm{Th}_{\min }$ using LAUC

End if

\section{SIMULATION}

In this section, we examine the performance of HMM based Traffic Prediction (HMM-TP) technique with an extensive simulation study based upon the ns-2 network simulator.

Topology considered for the simulation is a mesh topology depicted in Fig. 1. There are 14 edge nodes and 14 core nodes with a total of $101 \mathrm{Gbps}$ wavelength channels per link of which 2 are control channels and the rest 8 are data channels. Header-burst offset time of $40 \mu \mathrm{s}$ has been used for simulation.

Self-similar traffic model is used for simulating short-term traffic and TCP is used for long-term. 6 TCP traffic flows and 5 sets of short-term traffic flows are set up between each pair of ingress-egress nodes. Shortest path routing with available unused channel filling in each of the appropriate wavelength subgroups with deflection routing of contending bursts has been employed. The normalized load defined as the total actual load per link to the total capacity of the link has been varied from 0.1 to 1 . An offline analysis of connection holding times for probe traffic of varying normalized loads of short traffic along all paths of this network, monitored for a period of $10 \mathrm{~s}$, was used to calculate average connection holding times. This average connection time over all paths for short traffic is used to set threshold minLth. In order to reduce the transmission overheads which are higher for short bursts, the number of wavelength channels to be assigned for short term flows should be larger and hence $\mathrm{Th}_{\max }$ is set to be $3(0.37 \%$ of $\mathrm{N})$. The performance of the HMM based ABS-WA, is compared with the adaptive Optical Burst Switching (AOBS) method reported by (Bonald et al., 2011). For the HMM-TP simulation, out of 8 data channels considered, $\mathrm{Th}_{\min }$ was set at 3 resulting in 5 available channels for short-term traffic and 3 channels for longterm traffic. The minsize and maxsize values for the burst size are set as $10 \mathrm{~KB}$ and $40 \mathrm{~KB}$, respectively. 


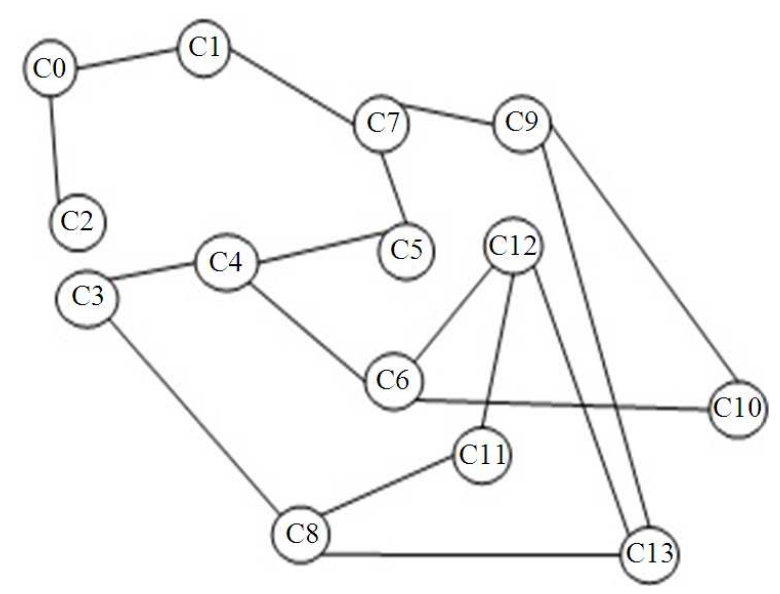

Fig. 1. Network topology

\section{RESULTS}

Simulations were carried out by varying the normalized traffic load from 0.1 to 1.0 and all the blocking probability, end-to-end delay, packets throughput ratio, was monitored for both AOBS and our HMM based ABS-WA schemes.

\subsection{Blocking Probability}

Figure 2 shows the blocking probability for ABSWA and AOBS techniques. As the normalized traffic load is increased from 0.1 to 1 , we can see that the blocking probability increases, because of congestion and overloading. The proposed ABS-WA technique has $42.7 \%$ lower blocking probability when compared to AOBS since it adaptively adjusts the burst size, based on holding time. In order to verify which kind of traffic shows lesser blocking probability, simulation results were analyzed for long traffic and short traffic separately.

The results of burst delay and burst received are depicted in Fig. 3a and b, respectively, for both the techniques. The overall burst delay is reduced by $0.38 \%$ and the received bursts are $10.4 \%$ more, for ABS-WA when compared to AOBS.

\subsection{Packet loss ratio and end to end delay}

Figure 4 shows that packet loss ratio for short traffic is lower by $27 \%$ and long traffic is lower by $62 \%$ when compared to AOBS technique. The reduction in the blocking probability of short traffic is attributed to the larger number of wavelengths allocated for the same.

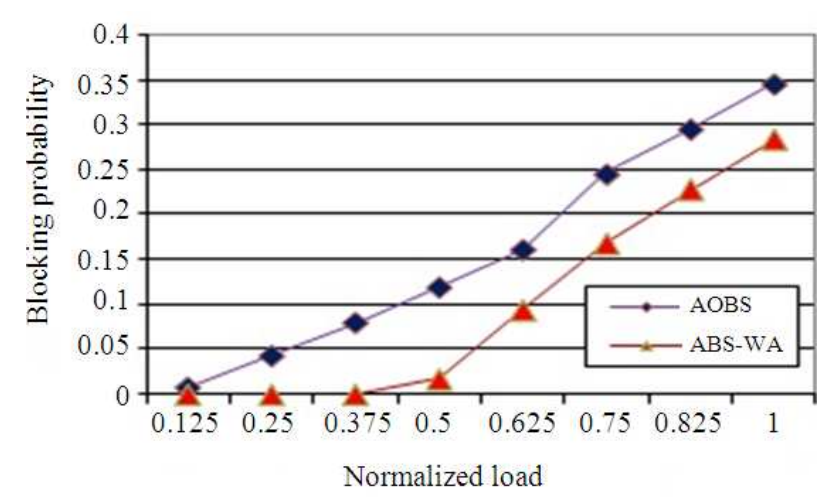

Fig. 2.Variation of Blocking Probability with normalized load

Even though number of wavelengths allocated for long traffic is lesser, the blocking probability for long traffic remains lower by 62 and $78 \%$ respectively in comparison to AOBS showing that this scheme provides more efficient use of wavelengths. AOBS allocates burst sizes based on total number of traffic flows in the network at a point of time and not on the load in the particular route whereas our technique allocates burst sizes based on connection time availability of the particular route at the particular instant of time and is hence more efficient.

In comparison to AOBS scheme, the reduction in average end-to-end delay in short traffic using HMM ABS-WA is only $0.02 \%$ but the throughput ratio is larger in accordance with the trend observed in blocking probability (Fig. 4). For long traffic, a large reduction in delay by $78 \%$ is seen for HMM ABS-WA scheme (Fig. 5) which is another consequence of connection-time aware provisioning of ABS-WA scheme in comparison to the AOBS scheme of fixed increase in burst size based on number of active flows in the network.

For short term traffic the end to end delay is almost similar between HMM-TP and AOBS but there is $8 \%$ increase in throughput. For long term traffic the end to end delay for traffic flows in HMM-TP is much less over AOBS and thereby results in higher throughput for the proposed traffic prediction. This is due to that AOBS assigns fixed increase on burst size based on number of active links in the network but HMM-TP actively predicts the resource utilization on links and assigns burst size accordingly. 


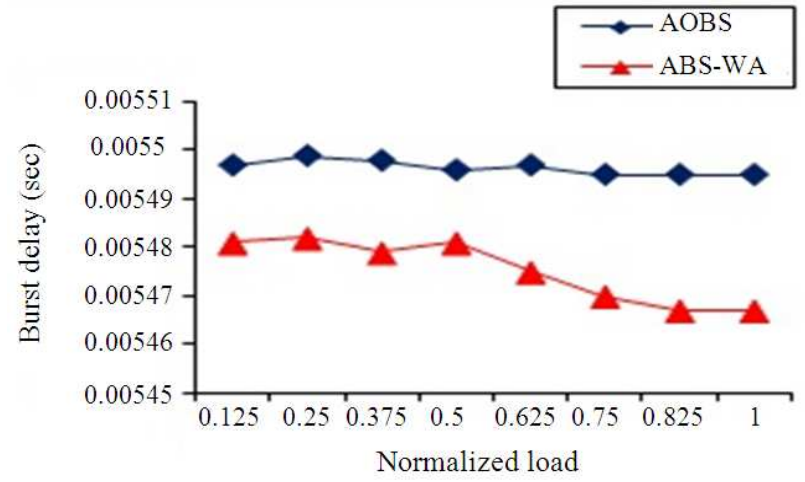

(a)

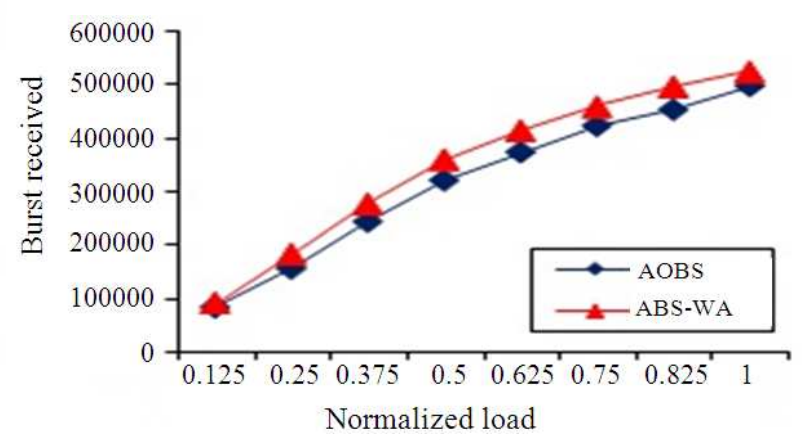

(b)

Fig. 3. (a) Normalized Load Vs Burst Delay (b) Normalized Load Vs Burst received

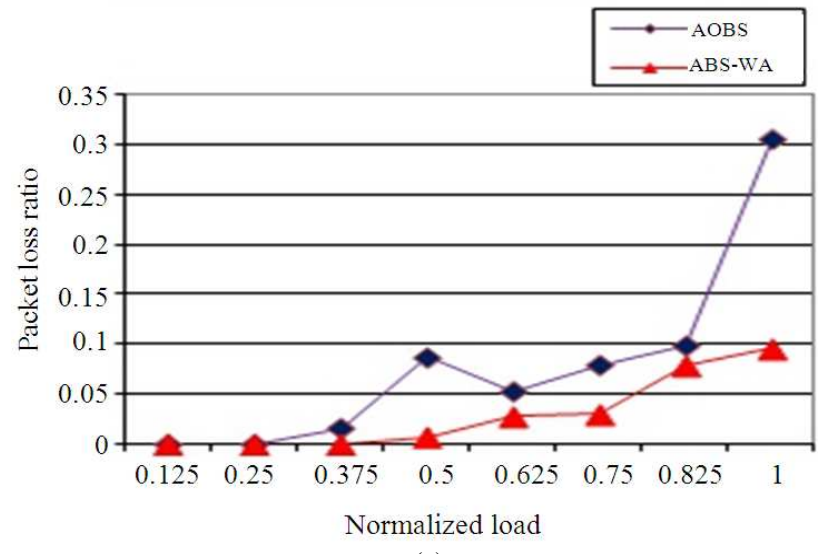

(a)

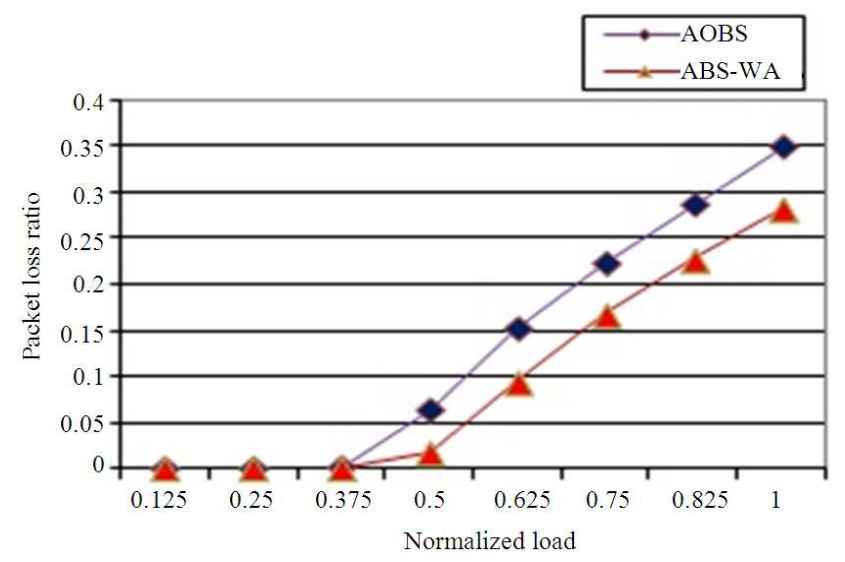

(b)

Fig. 4. Variation of loss ratio of packets with normalized load for (a) long traffic and (b) short traffic

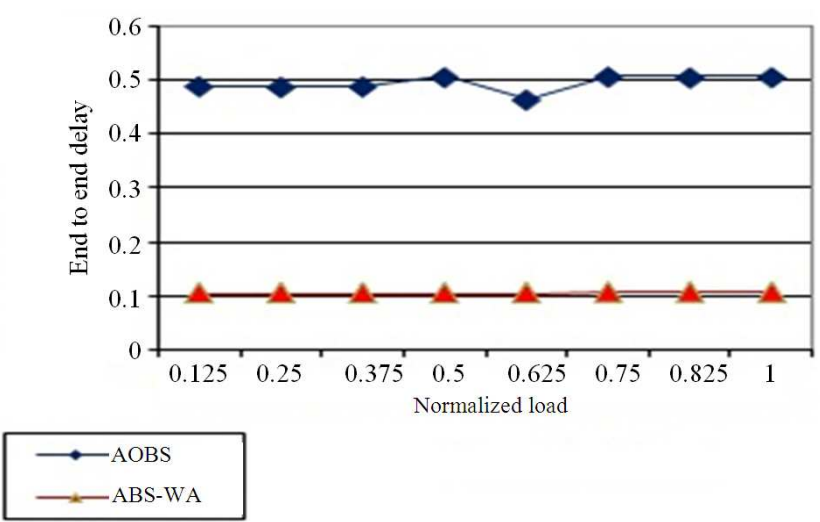

(a)



(b)

Fig. 5. End to end delay Traffic Characteristics for (a) long traffic (b) short traffic flows 


\section{CONCLUSION}

This study, reports a study of using HMM based traffic prediction for both wavelength allocation and adaptive burst sizing technique on OBS network. Our technique also uses deflection routing technique to transmit the bursts through the optical network. HMM predicted link holding time has been used for adaptive burst sizing and for classification into short term flows which are assigned a separate set of wavelengths with larger number of channels set aside for short-term traffic. This method effectively increases throughput and reduces blocking probability when compared to AOBS reported earlier (Bonald et al., 2011). The improvement in the performance metrics of short-term traffic is mainly due to allocation of larger number of wavelength channels whereas those of long traffic is attributed to connection time aware burst sizing in each route. The proposed HMM predicted ABS-WA resource allocation method based on link holding time reduces the burst collision rate by more than $40 \%$ in comparison to previous work (AOBS scheme) leading to concomitant decrease in delay and improvement in throughput. Segregation of wavelengths for short bursts allows fast switching schemes to be employed preferentially for these wavelengths and slow but energy saving MEMS switching for long bursts enabling hybrid switching schemes to be implemented in each node. If the delays and throughput of the short bursts need to be improved, then one alternative is to use higher bit-rates selectively on these wavelengths. These schemes along with hybrid loss recovery has to be investigated further. Adaptive variation of number of wavelengths assigned depending on the dynamic changes in the short and long traffic ratios and the available number of data channels for the same network also needs further investigation.

\section{REFERENCES}

Bonald, T., R. Indre and S. Oueslati, 2011. Adaptive optical burst switching. Proceedings of the 23rd International Teletraffic Congress, Sept. 6-9, IEEE Xplore Press, San Francisco USA, pp: 150-157.

Chomphan, S., 2012. Design of tree structure in context clustering process of hidden markov model-based thai speech synthesis. Am. J. Applied Sci., 9: 313320. DOI: $10.3844 /$ ajassp.2012.313.320
Coulibaly, Y., M.S.A. Latiff, A.B. Mohammad and A.M. Umaru, 2011. Priority-based time slot assignment algorithm for hierarchical time sliced optical burst switched networks. Proceedings of the 6th International Conference Systems Networks Communications, Oct. 23-29, Barcelona, Spain, pp: 199-205.

Dainotti, A., A. Pescape, P.S. Rossi, F. Palmieri and G. Ventre, 2008. Internet traffic modeling by means of Hidden Markov Models. Comput. Netw., 52: 26452662. DOI: 10.1016/j.comnet.2008.05.004

Drakos, A., T.G. Orphanoudakis and A. Stavdas, 2012. Performance benchmarking of core optical networking paradigms. Optics Express, 20: 1742117439. DOI: $10.1364 /$ OE.20.017421

Fiorani, M., M. Casoni and S. Aleksic, 2013. Hybrid optical switching for Energy efficiency and QoS differentiation in core networks. J. Opt. Comm. Netw., 5: 484-497. DOI:10.1364/JOCN.5.000484

Gerstel, O., M. Jinno, A. Lord and S.J.B. Yoo, 2012. Elastic optical networking: A new dawn for the optical layer. IEEE Commun. Mag., 50: s12-s20. DOI: 10.1109/MCOM.2012.6146481

Johnson, E.L., K.M. Sivalingam and M. Mishra, 2001. Scheduling in optical WDM networks using hidden Markov chain based traffic prediction. Phot. Netw. Commun., 3: 269-283. DOI: 10.1023/A:1011407532652

Khanna, R. and H. Liu, 2006. System approach to intrusion detection using hidden markov model. Proceedings of the International Conference Wireless Communications Mobile Computing, Jul. 03-06, Canada, pp: 349-354. DOI: 10.1145/1143549.1143619

Rabiner, L.R., 1989. A tutorial on hiddenmarkov models and selected applications in speech recognition. Proc. IEEE, 77: 257-286. DOI: 10.1109/5.18626

Ramantas, K. and K. Vlachos, 2011. A TCP prediction scheme for enhancing performance in OBS networks. Proceeding of the IEEE International Conference on Communications, (CC' 11), Jun. 5-9, IEEE Xplore Press, Kyoto, pp: 1-6. DOI: 10.1109/icc.2011.5962751

Rumley, S., O. Pedrola, M. Klinkowski, P. Pedroso and C. Gaumier et al., 2009. Adaptive burst admission and forwarding in obs networks. Proceedings of the IEEE 11th International Conference Transparent Optical Networks, Jun. 28-Jul. 2, IEEE Xplore Press, Azores, pp: 1-6. DOI: 10.1109/ICTON.2009.5185009 
Shekhawat, V.S., D.K. Tyagi and V.K. Chaubey, 2008. Weight based edge disjoint path routing and wavelength assignment (wedp-rwa) algorithm for wdm networks. Proceedings of the 3rd International Conference Industrial Information Systems, Dec. 810, IEEE Xplore Press, Kharagpur, pp: 1-5. DOI: 10.1109/ICIINFS.2008.4798409

Tornatore, M., A. Pattavina, J. Zhang, B. Mukherjee and C. Ou, 2005. PHOTO: An efficient shared-pathprotection strategy based on connection-holdingtime awareness. J. Lightwave Technol., 23: 31383146. DOI: $10.1109 /$ JLT.2005.856174
Zhang, J.Y., J. Wu, G.V. Bochmann and M. Savoie, 2009. Differentiated static resource allocation in WDM networks. Proceeding of the IEEE International Conference Communications, Jun. 1418, IEEE Xplore Press, Germany, pp: 1-5. DOI: 10.1109/ICC.2009.5198917 